\title{
Inhibition of prostaglandin E2 production by synthetic minor prenylated chalcones and flavonoids: synthesis, biological activity, crystal structure, and in silico evaluation
}

\begin{abstract}
The discovery of potent inhibitors of prostaglandin E2 (PGE2) synthesis in recent years has been proven to be an important game changer in pharmaceutical industry. It is known that excessive production of PGE2 triggers a vast array of biological signals and physiological events that contributes to inflammatory diseases such as rheumatoid arthritis, atherosclerosis, cancer, and pain. In this Letter, we report the synthesis of a series of minor prenylated chalcones and flavonoids which was found to be significantly active in suppressing the PGE2 production secreted by lipopolysaccharide-induced mouse macrophage cells (RAW 264.7). Among the compounds tested, 14b showed a dose-response inhibition of PGE2 production with an IC50 value of $2.1 \varepsilon \mathrm{M}$. The suppression upon PGE2 secretion was not due to cell death since $14 \mathrm{~b}$ did not reduce the cell viability in close proximity to the PGE2 inhibition concentration. The obtained atomic coordinates for the single-crystal XRD of 14b was then applied in the docking simulation to determine the potential important binding interactions with murine COX-2 and mPGES-1 putative binding sites.
\end{abstract}

Keyword: Prenylated chalcone; Minor flavonoids; Single-crystal XRD; Prostaglandin E2; COX-2 inhibitor; ADMET prediction 